\title{
Imaging response evaluation after novel neoadjuvant treatments of pancreatic cancer
}

\author{
Eva-Maria Gassner (D) Paulina Poskaite
}

Received: 14 February 2019 / Accepted: 29 March 2019 / Published online: 17 May 2019

(C) The Author(s) 2019

\begin{abstract}
Summary
Background Emergent neoadjuvant concepts have improved the rate of margin-free (R0) resections in borderline resectable and locally advanced pancreatic ductal adenocarcinoma. However, due to specific properties of pancreatic cancer, imaging response assessment is difficult.

Methods The concepts of histologic response patterns and their imaging correlates are outlined, together with efforts in morphologic and functional imaging to overcome the challenges of response assessment. Results Imaging evaluation after neoadjuvant therapy remains a diagnostic dilemma. To date, better understanding of the specific transformations of PDAC under neoadjuvant agents could not be transformed into validated imaging criteria for defining response and selecting surgical candidates.

Conclusion Currently, patients with non-metastatic borderline resectable and locally advanced pancreatic ductal adenocarcinoma are brought to diagnostic laparotomy in the absence of overt radiologic progression after neoadjuvant therapy. Apart from morphologic patterns, ongoing research is focused on defining robust functional imaging parameters for response assessment.
\end{abstract}

Keywords Computed Tomography · Magnetic Resonance Imaging - 18-Fluorodeoxyglucose PET . Pancreatic ductal adenocarcinoma $\cdot$ Neoajuvant therapy $\cdot$ Resectability

E.-M. Gassner, MD · P. Poskaite, MD $(\bowtie)$

Department of Radiology, Medical University Innsbruck, Anichstraße 35, 6020 Innsbruck, Austria

paulina.poskaite@i-med.ac.at

E.-M. Gassner, MD

eva-maria.gassner@i-med.ac.at

\begin{tabular}{|c|c|}
\hline ns & \\
\hline AHPBA/SSO/SSAT & $\begin{array}{l}\text { American Hepato-Pancreato-Bil- } \\
\text { iary Association/Society of Surgi- } \\
\text { cal Oncology/Society for Surgery } \\
\text { of the Alimentary Tract }\end{array}$ \\
\hline $\mathrm{BR}$ & Borderline resectable \\
\hline DWI & Diffusion-weighted imaging \\
\hline LA & Locally advanced \\
\hline MDCT & $\begin{array}{l}\text { Multi-detector computed tomog- } \\
\text { raphy }\end{array}$ \\
\hline MRI & Magnetic resonance imaging \\
\hline NAT & Neoadjuvant treatment \\
\hline NCNN & $\begin{array}{l}\text { National Comprehensive Cancer } \\
\text { Network }\end{array}$ \\
\hline PDAC & $\begin{array}{l}\text { Pancreatic ductal adenocarci- } \\
\text { noma }\end{array}$ \\
\hline $\mathrm{PET} / \mathrm{CT}$ & $\begin{array}{l}\text { 18-Fluorodeoxyglucose positron- } \\
\text { emission tomography/computed } \\
\text { tomography }\end{array}$ \\
\hline RECIST & $\begin{array}{l}\text { Response Evaluation Criteria of } \\
\text { Solid Tumors }\end{array}$ \\
\hline R0 & $\begin{array}{l}\text { Margin-negative/margin-free re- } \\
\text { section }\end{array}$ \\
\hline
\end{tabular}

\section{Introduction}

Several trials have investigated the effect of novel neoadjuvant regimens on the resectability of both borderline resectable (BR) and locally advanced (LA) pancreatic ductal adenocarcinoma (PDAC). However, specific histopathologic changes of PDAC under neoadjuvant treatment (NAT) preclude accurate depiction of viable tumor remnants with cross-sectional imaging. This overview summarizes current knowledge of PDAC response patterns on diagnostic imaging, recent approaches to estimate downstaging and optimize patient selection for surgery based on imaging. 
Table 1 NCCN guidelines version 3. 2017 criteria defining borderline resectable (BR) and locally advanced (LA) pancreatic adenocarcinoma

\begin{tabular}{|c|c|c|c|c|c|}
\hline \multicolumn{6}{|c|}{ Borderline resectable (BR) pancreatic adenocarcinoma } \\
\hline & \multicolumn{3}{|c|}{ Pancreatic head/uncinated process } & \multicolumn{2}{|c|}{ Pancreatic body/tail } \\
\hline Arterial & SMA: tumor contact $\leq 180^{\circ}$ & $\begin{array}{l}\text { CHA: tumor contact of } \\
\leq 180^{\circ} \text { without extension } \\
\text { to CA or hepatic artery } \\
\text { bifurcation allowing for re- } \\
\text { section and reconstruction }\end{array}$ & Contact with variant artery & \multicolumn{2}{|c|}{$\begin{array}{l}\text { CA: contact of } \leq 180^{\circ} \text { or contact of }>180^{\circ} \text { with- } \\
\text { out involvement of the aorta and with intact GDA } \\
\text { permitting a modified Appleby procedure }\end{array}$} \\
\hline Venous & \multicolumn{3}{|c|}{$\begin{array}{l}\text { Tumor contact with the SMV or } \mathrm{PV} \text { of }>180^{\circ} \text {, or contact of } \leq 180^{\circ} \text { with contour irregularity } \\
\text { or vein thrombosis, but with suitable vessel proximal and distal for reconstruction }\end{array}$} & \multicolumn{2}{|l|}{ Tumor contact with the IVC } \\
\hline \multicolumn{6}{|c|}{ Locally advanced (LA) pancreatic adenocarcinoma } \\
\hline & \multicolumn{3}{|c|}{ Pancreatic head/uncinated process } & \multicolumn{2}{|c|}{ Pancreatic body/tail } \\
\hline Arterial & Tumor contact with SMA $>180^{\circ}$ & $\begin{array}{l}\text { Tumor contact with the CA } \\
>180^{\circ}\end{array}$ & $\begin{array}{l}\text { Tumor contact with the } \\
\text { first jejunal SMA branch }\end{array}$ & $\begin{array}{l}\text { Tumor contact of }>180^{\circ} \\
\text { with the SMA or CA }\end{array}$ & $\begin{array}{l}\text { Tumor contact with } \\
\text { the CA and aorta }\end{array}$ \\
\hline Venous & $\begin{array}{l}\text { Unreconstructible SMV/PV due } \\
\text { to tumor involvement or oc- } \\
\text { clusion due to tumor or bland } \\
\text { thrombus }\end{array}$ & $\begin{array}{l}\text { Contact with most prox- } \\
\text { imal draining jejunal } \\
\text { branch into SMV }\end{array}$ & - & $\begin{array}{l}\text { Unreconstructible SMV/PV } \\
\text { due to tumor involvement } \\
\text { or occlusion due to tumor } \\
\text { or bland thrombus }\end{array}$ & - \\
\hline \multicolumn{6}{|c|}{ Distant metastasis including non-regional lymph nodes } \\
\hline
\end{tabular}

Non-metastatic pancreatic adenocarcinoma is categorized into three surgical stages at the time of diagnosis: resectable $(\mathrm{R})$, borderline resectable $(\mathrm{BR})$, and locally advanced (LA) [1]. Currently, several classification systems are used to define resectability with a required safety margin of $>1 \mathrm{~mm}$. The consensus definitions published by the NCNN/AHPBA/SSO/SSAT, as well as definitions of resectability from the MD Anderson Cancer Centre are most widely used ([2, 3]; Ta-

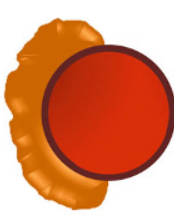

a

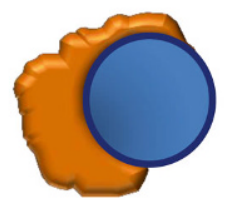

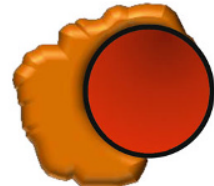

b

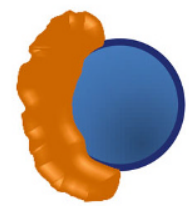

d

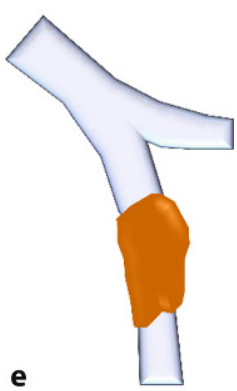

Fig. 1 Schematic representations of the tumor-vessel contact. Images correspond with the National Comprehensive Cancer Network NCNN definitions of resectability in Table 1. a Arterial tumor contact of $\leq 180^{\circ}$ and $\mathbf{b}$ of $>180^{\circ}$. The 180degree threshold of arterial involvement denotes the cut-off between borderline resectable and non-resectable/locally advanced disease in patients with adenocarcinoma of the pancreatic head and uncinated process. c-e Portal venous and superior mesenteric vein involvement with c, contact of $>180^{\circ}$ or d, contact of $\leq 180^{\circ}$ with contour irregularity or thrombosis is considered borderline resectable, when proximal and distal vessel margins are amenable to reconstruction (as shown in e) ble 1 and Fig. 1). Since the recognition of borderline resectability in 2008 [4], neoadjuvant concepts have been implemented to downstage patients from either BR or LA-PDAC to a more favorable surgical stage in order to achieve curative surgery ([5]; Fig. 2).

Gemcitabine-based regimens, recently combined with nab-paclitaxel [6], and the advent of FOLFIRINOX-based schemes in 2011 [7], both with or without additional chemoradiation, hold promise for downstaging PDAC and more importantly, enhance the rate of R0 resection $[8,9]$.

Unfortunately, response evaluation on imaging and prediction of resectability have proven challenging due replacement of tumor by fibrosis $[10,11]$. Although biomarker levels and clinical performance status are incorporated into reassessment [1], multiphase contrast-enhanced multidetector CT (MDCT) with three-dimensional reconstructions remains the mainstay for further patient selection for surgery [12]. Meanwhile, advances in magnetic resonance imaging (MRI), notably faster acquisition techniques and enhanced image quality due to improved signal-tonoise and contrast-to-noise ratios, have brought MRI close to the spatial resolution of MDCT, while offering superior contrast resolution and the opportunities of functional imaging in terms of diffusion-weighted imaging (DWI).

\section{Histopathologic response patterns of PDAC}

It was recognized early that NAT-induced tumor cell injury in PDAC is mainly reflected by "isovolumetric" tissue replacement through fibrosis, rather than volume loss. In 1992, Evans at al. reported the histologic changes in 17 resected specimens after NAT and established a pathologic response grading sys- 


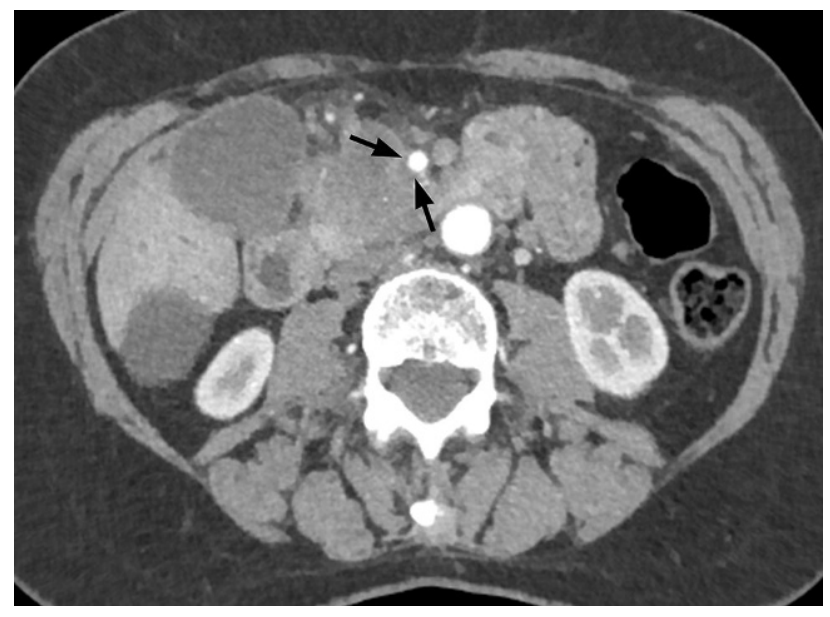

Fig. 2 CT image showing a contact of $\leq 180^{\circ}$ between tumor and the superior mesenteric artery (arrows), defined as borderline resectable disease according to the National Comprehensive Cancer Network (NCCN) version 2017 guidelines

tem for PDAC [13] by describing the percentage of viable tumor cells within the specimen. In their review, Kalimuthu et al. outlined histopathologic changes after NAT in more detail [14], with emphasis on subtle residual tumor nests scattered throughout the fibrotic tissue.

A small percentage of a complete pathologic response ( $2 \%$ to $7 \%)$ was also reported in meta-analyses [15, 16]. Finally, patients with NAT are less likely to have positive lymph nodes and perineural invasion on histopathologic workup [17].

\section{Imaging response assessment with RECIST1.1} (clinical stage)

The concept of tissue replacement, along with perivascular/perineural patterns of growth, makes PADC ineligible for follow-up with RECIST 1.1. It is thought to be the cause of the repeatedly documented failure to predict viable tumor nests around crucial surgical structures using MDCT or MRI.

In their meta-analysis, Dhir et al. calculated overall RECIST1.1 response rates after NAT in a subset of 61 studies [18]. Overall, partial response was seen in $20 \%$ of patients, most patients remained stable $(59 \%)$, and progressive disease occurred in $16 \%$ of patients.

Pathologic response grading revealed an overall Evans grade III rate (tumor destruction of $>90 \%$ ) of $12 \%$. Grade IIB (tumor destruction of $51-90 \%$ ) was seen in $27 \%$, and $37 \%$ had grade IIA (11-50\% tumor destruction). Grade I ( $<10 \%$ tumor destruction) was found in $13 \%$ of specimens.

Thus, the majority of patients were stable or in partial response on cross-sectional imaging, and most of the resected specimens showed minor to moderate histologic response. However, as Xia [19] et al. demonstrated, radiologic and pathologic grading are not correlated on a patient basis (Fig. 3). In their work- up of 38 specimens after NAT, all pathologic response grades from minor to complete were present in both radiologic partial responders as well as in patients with stable disease. Minor pathologic response $(<$ than $50 \%$ tumor destruction) was dominant in the group of RECIST stable disease. There were three pathologic complete responders in their group, two with RECIST stable disease, and one with partial response.

\section{Imaging response assessment of surgical stage (resectability)}

With the advent of novel NAT concepts, such as FOLFIRINOX or gemcitabine/nab-paclitaxel, several groups have investigated radiologic tumor changes after therapy in recent years, with special attention to resectability. Meta-analyses initially suggested high R0 rates in patients with BR-PDAC: when $63 \%$ of patients were selected for surgery after FOLFIRINOX, R0 margins resulted in 93\% of them [20]. Patient selection becomes even more important in locally advanced PDAC: when $28 \%$ of patients were eligible for surgery after FOLFIRINOX, free margins were seen in $74 \%$ of operated patients [21]. On an intention-totreat basis, however, overall R0 margins resulted in only $22 \%$ of treated LA-PDAC patients [22]. A single recent study, however, reported a far higher R0 rate of $62 \%$ [22].

Generally, imaging workup after NAT reveals an overestimation of residual tumor burden and the probability of R0 resection is difficult to estimate (Fig. 4). Diagnostic accuracy for predicting resectability after NAT has yielded conflicting results so far: accuracy was set at $58 \%$ by a French group [23], whereas Kim et al., in an older publication, reported a high accuracy of $83 \%$ [24].

The effect of FOLFIRINOX on imaging resectability criteria compared to pathologic margins was presented in two publications with similar results:

Ferrone and colleagues found a shift to more advantageous resection categories on $\mathrm{CT}$ in a collective of 40 patients after FOLFIRINOX. While 19 patients still remained in the LA group radiographically, R0 margins were achieved in 35 patients (92\%) [25].

A French multicenter study by Wagner et al. [26] noted radiologic downstaging of resectability according to NCCN criteria in only 7/36 patients after FOLFIRINOX, while the majority of patients remained stable on imaging. However, R0 resections were achieved in 31 patients (86\%), among them 6 patients with persistent LA disease on CT imaging.

In this light, several studies have focused on imaging predictors for resectability and outcome, with heterogeneous approaches and results. 


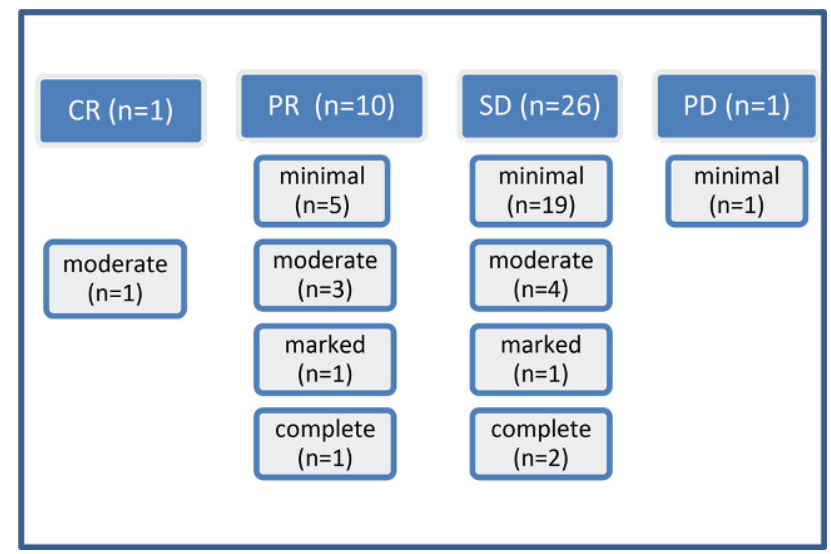

Fig. 3 Imaging response using RECIST (Response Evaluation Criteria In Solid Tumors) version 1.1, and Evans pathologic response grades in pancreatic specimen found by Xia et al. [19]. RECIST classification: $C R$ complete response, $P R$ partial response, $S D$ stable disease, $P D$ progressive disease. Pathologic response grades: minimal, $<50 \%$ tumor destruction; moderate, 50-90\% tumor destruction, marked, $>90$ tumor destruction; complete, no tumor cells

\section{Regression of vessel contact was explored in two studies}

Cassinotto et al. found regressive circumferential tumor contact with crucial vessels (superior mesenteric vein, portal vein, and superior mesenteric artery) being highy predictive for R0 resections (PPV: 91\%). Specifically, a regression in SMA contact yielded an odds ratio of 3.82 (95\% CI: 1.27-11.5) for free margins [27]. However, no correlation between persistent narrowing of SMV/PV and R0 resection was observed.

Similarly, in the publication by Wagner [26], regressive circular vascular involvement, both arterial and venous (PV and SMV), resulted in longer disease-free survival than stable circumferential contact.

\section{Size and resectability}

Michelakos et al. confirmed tumor size after FOLFIRINOX (median $2.3 \mathrm{~cm}$ in resected group vs. $3 \mathrm{~cm}$ in non-resected) to be associated with an attempt for resection at surgical exploration (R0 in the resected group: $80 \%$ ), which is consistent with other studies $[27,29]$. Changes in size were not predictive for resectability [28].

\section{Tumor enhancement}

Marchegiani et al., similar to prior studies [26, 27], observed an increase in contrast uptake in pancreatic tumors after NAT [29], attributed to fibrosis. Enhancement in their study was significantly higher in R0-resected cancer. However, in the publications by Wagner [26] and Casinotto [27], neither absolute enhancement nor enhancement changes after NAT were correlated to $\mathrm{R} 0$ resection.

In most studies, patients proceeded to resection when they had stable disease or partial response on imaging, while non-resected patients were excluded, which potentially distorts the comparison of imaging features to histologic margins. Furthermore, all studies were retrospective in design, and apart from the French multicenter study, all were single center.

While most studies were set up with two readers in consensus or with high interobserver concordance, interestingly, a study in 2010 presented inter-reader agreement for predicting resectability as an endpoint. A multi-rater kappa value of no more than 0.28 was calculated for predicting resectability after NAT, owed mainly to a high variability of sensitivities, ranging from 14 to $86 \%$. However, the sample size was small with only seven resectable patients after NAT.

\section{Does cross-sectional imaging always overesti- mate viable tumor?}

While the majority of papers suggest an overestimation of the viable tumor remnant, Amodeo et al. re-
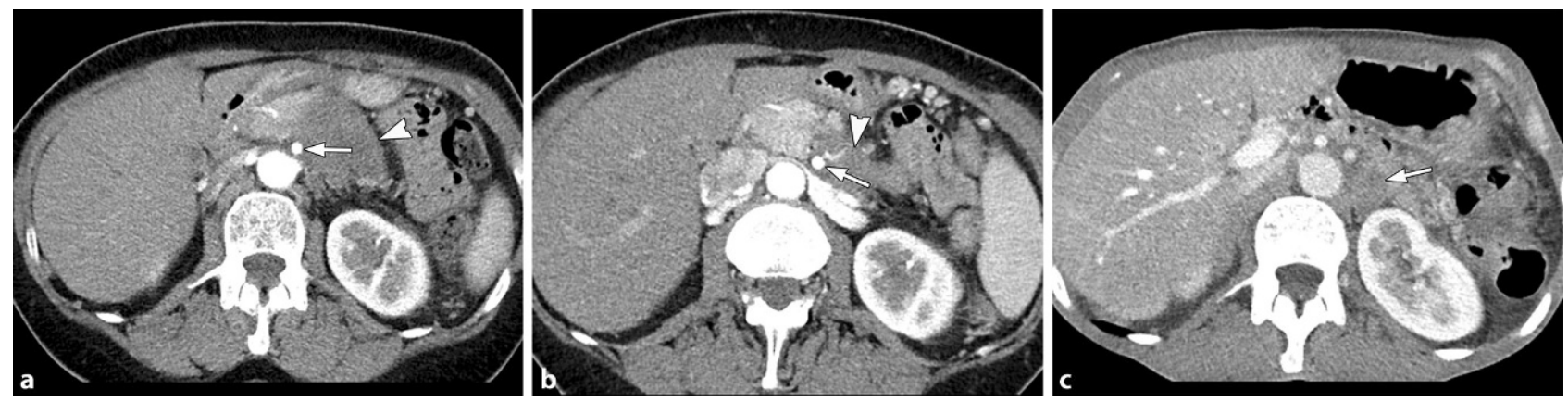

Fig. 4 a Locally advanced PDAC in a 52-year-old female at baseline (arrowhead), with encasement of the superior mesenteric artery of $>180^{\circ}$ (arrow). b The same patient after neoadjuvant therapy with FOLFIRINOX. Tumor shrinkage can be seen (arrowhead); however, SMA encasement persists (arrow). The patient underwent total pancreatectomy with negative resection margins. c Local recurrence 1 year after total pancreatectomy in the same patient (arrow) 

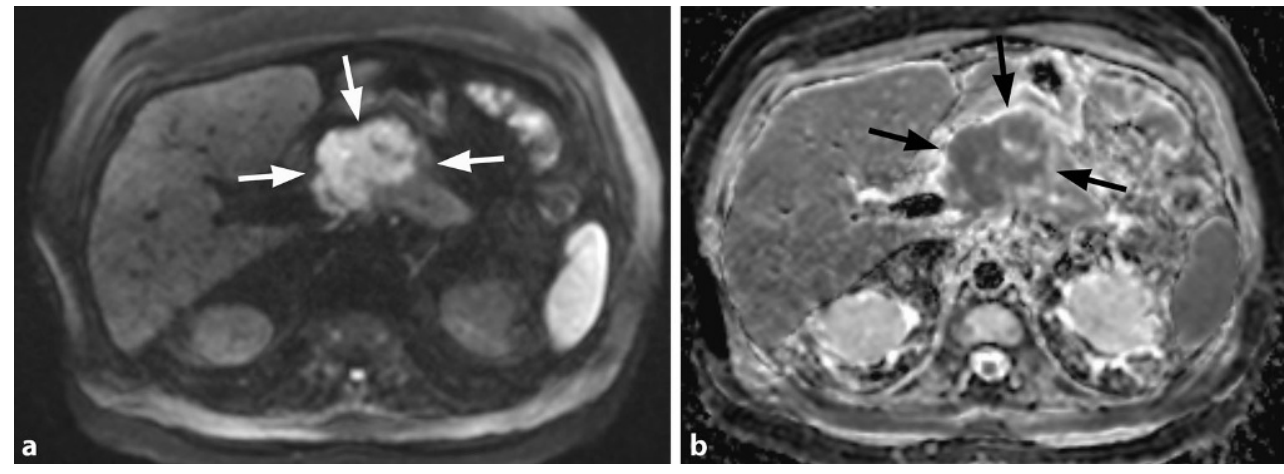

Fig. 5 a Diffusion weighted image (DWI) of a PDAC at a $b$ value of $800 \mathrm{~s} / \mathrm{mm}^{2}$ reveals high signal intensities (arrows) as markers of restricted water diffusion within the tumor. b Corresponding ADC map shows an inverted image with low signal

ported an underestimation in four out of seven patients who were classified as RECIST responders but had stable disease at laparotomy [30]. Also, with regard to NCCN resectability, cross-sectional imaging can occasionally underestimate the true extent of tumor: in the study by Marchegiani, seven patients were deemed resectable after NAT, but only five of them had $\mathrm{R} 0$ resection.

Finally, the true extent of tumor within fibroinflammatory tissue replacement has not been systematically explored so far with radiologic-pathologic lesionby-lesion work-up.

\section{Functional imaging: 18-FDG PET and diffusion- weighted MRI}

18-FDG-PET is established in the response evaluation of lymphoma [31] and seems superior to morphologic response assessment in neoadjuvant therapy of esophageal cancer [32].

PERCIST1.0 criteria use a lean-body-mass-normalized standard uptake value (SUL) to compare peak values before and after therapy [33]. On the other hand, traditional parameters, such as maximum standardized uptake value (SUVmax) and body-weightnormalized SUV (SUVbw), are still in use to compare peak metabolic activities before and after NAT.

In a recent publication comprising 14 subjects and comparing both RECIST 1.1 and PERCIST1.0 criteria to pathologic residual tumor, an insignificant correlation of $r=0.26$ was found between PERCIST and pathology, and none between RECIST and pathology. With known inadequacy of RECIST1.1, the limited but additional value of PERCIST was highlighted [34].

Prior studies with PET/CT similarly yielded ambiguous results. Mellon et al. [35] found a correlation between post-NAT SUVmax and pathologic response grade, but no correlation with the decrease of SUVmax. Of note, seven out of 81 patients with BRor LA-PDAC were excluded from the study, since PET failed to show any detectable baseline SUVmax. intensities in pathologic areas (arrows). ADC maps are used to quantify diffusion restriction with manufacturer-specific software computation

A Japanese group confirmed a high variability of SUVmax at baseline and post NAT, as well as a high variability of regression indices [36]. For statistics, values were dichotomized along their median. Doing so, SUVmax after NAT was not associated with response, a result contrary to the work of Mellon, while baseline SUVmax and regression index were.

Diffusion-weighted MR imaging (DWI) offers functional tissue assessment by mapping the restriction of Brownian water molecule motion. Diffusion restriction is a marker for cellularity and pathologic characteristics of cellular barriers, both increased in tumors [37]. Calculating the apparent diffusion coefficient (ADC) in multiples of $10^{-3} \mathrm{~mm}^{2} / \mathrm{sec}$ from diffusionweighted images allows for quantitative assessment of restricted diffusion. As such, on ADC maps, low values-depicted as dark areas-represent restricted diffusion (Fig. 5). ADC maps are widely investigated in oncologic imaging to estimate treatment response with promising results [38, 39].

Although Cuneo and colleagues first found a high correlation between ADC values and pathologic response in a small group of 7 patients [40], the variability of absolute pretreatment and posttreatment ADC values and their changes proved challenging. Okada et al. found pretreatment ADC values to be more indicative of response and surgical margins than posttreatment values and ADC changes. As another result of their study, MRI-ADC parameters outperformed the respective PET-SUVmax values [41].

In an analysis of 23 patients, however, Dalah et al. presented posttreatment ADC values and pathologic response to be moderately correlated $(\mathrm{r}=0.517$, $p=0.02$ ) [42]. The study is notable for using elaborate postprocessing tools such as color-coded ADC maps and histograms to demonstrate tumor heterogeneity after NAT.

Both publications pointed in the same direction by highlighting a significant difference between pre- and posttreatment mean ADC values.

Contrary to PET, which requires an interval of at least 8 weeks between NAT and restaging, in order 
to allow restitution of actinic inflammation, MRI was performed within 3-5 weeks after completing NAT.

Both functional methods may allow for estimating the overall response of a mass and predict survival $[37,43]$, but they lack the spatial resolution to predict surgical margins around vessels. Still, in the light of evidence that pathologic response grade might be a factor associated with survival, biomarker imaging may play a role in future decision algorithms.

\section{Conclusion}

The NCNN and ESMO panels currently endorse neoadjuvant therapy in borderline resectable PDAC $[44,45]$ and several individual studies have shown that in a minority of patients, NAT enabled R0 resection of locally advanced PDAC. However, on an intention-totreat basis, according to meta-analyses, the overall R0 rate was only about $54 \%$ in BR-PDAC [20] and $22 \%$ in LA-PDAC [22].

This poses a considerable challenge to interdisciplinary teams seeking to identify patients with potentially resectable disease. Histologic work-up suggests an inhomogeneous response of PDAC with interspersed tumor nests within fibrotic changes.

The current consensus is that radiologic mass regression occurs in a minority, while most patients remain stable on imaging. Generally, cross-sectional imaging overestimates the amount of residual viable tumor around vessels and thus cannot predict resectability.

There is no consistency across studies with regard to predictive imaging parameters. Most studies are retrospective, single-center, observational studies, examining a range of hypotheses, such as changes in tumor size, vessel contact, or enhancement. Due to heterogeneity, results are non-comparable; furthermore, statistical power is limited by small sample sizes.

At present, guidelines recommend taking patients to surgery after NAT when there is no tumor progression on cross-sectional imaging.

Functional imaging such as 18-FDG-PET/CT and MR-DWI reveal conflicting results in the search for optimal imaging predictors of response. But while markers of low cellularity may be indicators of response, to date, functional imaging methods lack the spatial resolution to detect microscopic disease at the crucial interface between mass and vessel wall. It is thought that this limit will be overcome in the years to come.

Funding Open access funding provided by University of Innsbruck and Medical University of Innsbruck.

Conflict of interest E.-M. Gassner and P. Poskaite declare that they have no competing interests.

Open Access This article is distributed under the terms of the Creative Commons Attribution 4.0 International License (http://creativecommons.org/licenses/by/4.0/), which permits unrestricted use, distribution, and reproduction in any medium, provided you give appropriate credit to the origi- nal author(s) and the source, provide a link to the Creative Commons license, and indicate if changes were made.

\section{References}

1. Evans DB, George B, Tsai S. Non-metastatic pancreatic cancer: resectable, borderline resectable, and locally advanced-definitions of increasing importance for the optimal delivery of multimodality therapy. Ann Surg Oncol. 2015;22:3409-13.

2. Tempero MA, Malafa MP, Al-Hawary M, et al. Pancreatic adenocarcinoma, version 2.2017, NCCN clinical practice guidelines in oncology. J Natl Compr Canc Netw. 2017;15(8):1028-61.

3. Reynolds RB, Folloder J. Clinical management of pancreatic cancer. J Adv Pract Oncol. 2014;5(5):356-64.

4. Katz MH, Pisters PW, Evans DB, et al. Borderline resectable pancreatic cancer: the importance of this emerging stage of disease. JAm Coll Surg. 2008;206:833-46.

5. Sadot E, Doussot A, O'Reilly EM, et al. FOLFIRINOX induction therapy for stage 3 pancreatic adenocarcinoma. Ann Surg Oncol. 2015;22(11):3512-21.

6. Von Hoff DD, Ervin T, Arena FP, et al. Increased survival in pancreatic cancer with nab-paclitaxel plus gemcitabine. NEngl J Med. 2013;369(18):1691-703.

7. Conroy T, Desseigne F, Ychou M, et al. Groupe Tumeurs Digestives of Unicancer, PRODIGE intergroup. FOLFIRINOX versus gemcitabine for metastatic pancreatic cancer. NEngl J Med. 2011;364(19):1817-25.

8. Suker M, Beumer BR, Sadot E, et al. FOLFIRINOX for locally advanced pancreatic cancer: a systematic review and patient-level meta-analysis. Lancet Oncol. 2016;17(6):801-10.

9. Amodeo S, Masi A, Melis M, Ryan T. Can we downstage locally advanced pancreatic cancer to resectable? A phase I/II study of induction oxaliplatin and 5-FU chemoradiation. J Gastrointest Oncol. 2018;9(5):922-35.

10. Katz MH, et al. Response of borderline resectable pancreatic cancer to neoadjuvant therapy is not reflected by radiographicindicators. Cancer. 2012;118(23):5749-56.

11. Morgan DE, Waggoner CN, Canon CL, et al. Resectability of pancreatic adenocarcinoma in patients with locally advanced disease downstaged bypreoperativetherapy: achallenge for MDCT. AJR Am J Roentgenol. 2010;194:615-22.

12. Lee ES, Lee JM. Imaging diagnosis of pancreatic cancer: a state-of-the-art review. World J Gastroenterol. 2014;20:7864-77.

13. Evans DB, Rich TA, Byrd DR, et al. Preoperative chemoradiation and pancreaticoduodenectomy for adenocarcinoma of the pancreas. Arch Surg. 1992;127:1335-9.

14. Kalimuthu SN, et al. The spectrum of histopathological changes encountered in pancreatectomy specimens after neoadjuvant chemoradiation, including subtle and lesswell-recognised changes. JClin Pathol. 2016;69:463-71.

15. Tang K, Lu W, Qin W, Wu Y. Neoadjuvant therapy for patients with borderline resectable pancreatic cancer: a systematic review and meta-analysis of response and resection percentages. Pancreatology. 2016;16(1):28-37.

16. Rombouts SJ, Walma MS, Vogel JA, et al. Systematic review of resection rates and clinical outcomes after FOLFIRINOXbased treatment in patients with locally advanced pancreatic cancer. Ann Surg Oncol. 2016;23(13):4352-60.

17. Schorn S, Demir IE, Reyes CM. The impact of neoadjuvant therapy on the histopathological features of pancreatic ductal adenocarcinoma-A systematic review and metaanalysis. Cancer Treat Rev. 2017;55:96-106. 
18. Dhir M, Malhotra GK, Sohal DPS, et al. Neoadjuvant treatment of pancreatic adenocarcinoma: a systematic review and meta-analysis of 5520 patients. World J Surg Oncol. 2017;15(1):18.

19. Xia BT, Fu B, WangJ, etal. Does radiologic response correlate to pathologic response in patients undergoingneoadjuvant therapy for borderline resectable pancreatic malignancy? JSurg Oncol. 2017;115(4):376-83.

20. Petrelli F, Coinu A, Borgonovo K, et al. FOLFIRINOXbased neoadjuvant therapy in borderline resectable or unresectable pancreatic cancer: a meta-analytical review of published studies. Pancreas. 2015;44(4):515-21.

21. Suker M, Beumer BR, Sadot E. FOLFIRINOX for locally advanced pancreatic cancer: a systematic review and patientlevel meta-analysis. Lancet Oncol. 2016;17(6):801-10.

22. Sherman WH, Hecht E, Leung D, Chu K. Predictors of response and survival in locally advanced adenocarcinoma of the pancreas following neoadjuvant GTX with or without radiation therapy. Oncologist. 2018;23(1):4-e10.

23. Cassinotto C, Cortade J, Belleannee G, etal. An evaluation of the accuracy of CT when determining resectability of pancreatichead adenocarcinoma after neoadjuvant treatment. Eur J Radiol. 2013;82:589-93.

24. Kim YE, Park MS, Hong HS, et al. Effects of neoadjuvant combined chemotherapy and radiation therapy on the CT evaluation of resectability and staging in patients with pancreatichead cancer. Radiology. 2009;250:758-65.

25. Ferrone C, Marchegiani G, Hong TS, et al. Radiological and surgical implications of neoadjuvant treatment with FOLFIRINOX for locally advanced and borderline resectable pancreatic cancer. Ann Surg. 2015;261:12-7.

26. Wagner M, Antunes C, Pietrasz D, et al. CT evaluation after neoadjuvant FOLFIRINOX chemotherapy for borderline and locally advanced pancreatic adenocarcinoma. Eur Radiol. 2017;27:3104-16.

27. Cassinotto C, Mouries A, Lafourcade JP. Locally advanced pancreatic adenocarcinoma: reassessment of response with CT after neoadjuvant CRT. Radiology. 2014;273(1):108-16.

28. Michelakos T, Pergolini I, Castillo CF. Predictors of resectability and survival in patients with borderline and locally advanced pancreatic cancer who underwent neoadjuvant treatment with FOLFIRINOX. Ann Surg. 2019;269(4):733-740

29. Marchegiani G, Todaro V, Boninsegna E. Surgery after FOLFIRINOX treatment for locally advanced and borderline resectable pancreatic cancer: increase in tumor attenuation on CT correlates with R0 resection. Eur Radiol. 2018;28(10):4265-73.

30. Amadeo S, Masi A, Mekis M. can we downstage locally advanced pancreatic cancer to resectable? A phase I/II study of induction oxaliplatin and 5-FU chemoradiation. J Gastrointest Oncol. 2018;9(5):922-35.

31. Cheson BD, Pfistner B, Juweid ME, et al. International harmonization project on Lymphoma. Revised response criteria for malignant lymphoma. J Clin Oncol. 2007;25(5):579-86.

32. Lordick F, Ott K, Krause BJ. PET to assess early metabolic response and to guide treatment of adenocarcinoma of the esophagogastric junction: the MUNICON phase II trial. Lancet Oncol. 2007;8:797-805.

33. Wahl RL, Jacene H, Kasamon Y, Lodge M. From RECIST to PERCISIT: evolving considerations for PET response criteria in solid tumors. J Nucl Med. 2009;50:112S-50S.

34. Dalah E, Tai A, Oshima K, et al. PET-based treatment response assessment for neoadjuvant chemoradiation in pancreatic adenocarcinoma: an exploratory study. Transl Oncol. 2018;11(5):1104-9.

35. Mellon EA, Jin WH, Frakes JM, et al. Predictors and survival for pathologic tumor response grade in borderline resectable and locally advanced pancreatic cancer treated with induction chemotherapy and neoadjuvant stereotactic body radiotherapy. Acta Oncol. 2017;56(3):391-7.

36. Kittaka H, Takahashi H, Ohigashi H. Role of (18)F-fluorodeoxyglucose positron emission tomography/computed tomographyin predicting the pathologicresponsetopreoperative chemoradiation therapy in patients with resectable T3 pancreatic cancer. World J Surg. 2013;37(1):169-78.

37. Thoeny HC, Ross BD. Predicting and monitoring cancer treatment response with DW-MRI. J Magn Reson Imaging. 2010;32(1):2-16.

38. Amodeo S, Rosman AS, Desiato V, et al. MRI-based apparent diffusion coefficient for predicting pathologic response of rectal cancer after neoadjuvant therapy: systematic review and meta-analysis. AJR Am J Roentgenol. 2018;211(5):W205-W16.

39. ACRIN 6698 Trial Team and I-SPY 2 Trial Investigators, Partridge SC, ZhangZ, Newitt DC, et al. Diffusion-weighted MRI findings predict pathologic response in neoadjuvant treatment of breast cancer: the ACRIN 6698 multicenter trial. Radiology. 2018;289(3):618-27.

40. Cuneo KC, Chenevert TL, Ben-Josef E, et al. A pilot study of diffusion-weighted MRI in patients undergoing neoadjuvant chemoradiation for pancreatic cancer. Transl Oncol. 2014;7:644-9.

41. Okada KI, Hirono S, Kawai M, et al. Value of apparent diffusion coefficient prior to neoadjuvant therapy is a predictor of histologic response in patients with borderline resectable pancreatic carcinoma. J Hepatobiliary Pancreat Sci. 2017;24(3):161-8.

42. Dalah E, Erickson B, Oshima K, et al. Correlation of ADC with pathological treatment response for radiation therapy of pancreatic cancer. Transl Oncol. 2018;11(2):391-8.

43. Kurosawa J, Tawada K, Mikata R, et al. Prognostic relevance of apparent diffusion coefficient obtained by diffusionweighted MRI in pancreatic cancer. J Magn Reson Imaging. 2015;42(6):1532-7.

44. National Comprehensive Cancer Network. NCCN clinical practice guidelines in oncology. Pancreatic adenocarcinoma. Version 2.2017. J Natl Compr Canc Netw. 2017;15(8):1028-61.

45. Ducreux M, Cuhna AS, Caramella C, et al. Cancer of the pancreas: ESMO Clinical Practice Guidelines for diagnosis, treatment and follow-up. Ann Oncol. 2015;26(Suppl 5):v56-v68.

Publisher's Note Springer Nature remains neutral with regard to jurisdictional claims in published maps and institutional affiliations. 